\title{
Ecomorfologia refletindo a dieta dos peixes em um reservatório no sul do Brasil
}

\author{
Ivan Teixeira ${ }^{1,3}$ \& Sirlei Terezinha Bennemann ${ }^{2}$
}

Biota Neotropica $v 7$ (n2) - http://www.biotaneotropica.org.br/v7n2/pt/abstract?article+bn00807022007

\author{
Recebido em 14/10/05 \\ Versão Reformulada recebida em 28/08/06 \\ Publicado em 01/05/07
}

${ }^{1}$ Curso de Pós-graduação em Ciências Biológicas (Mestrado), Departamento de Biologia Animal e Vegetal, Centro de Ciências Biológicas, Universidade Estadual de Londrina, CEP 86051-970, Londrina, PR, Brasil

${ }^{2}$ Departamento de Biologia Animal e Vegetal, Centro de Ciências Biológicas - CCB,

Universidade Estadual de Londrina, Rodovia Celso Garcia Cid (PR 445), Km 380,

CEP 86051-990, Londrina, PR, Brasil,e-mail: sirlei@uel.br

${ }^{3}$ Autor para correspondência: Ivan Teixeira,e-mail: geophagus@gmail.com

\begin{abstract}
Teixeira, I. \& S.T. Bennemann. Ecomorphology reflect the fish diet in a reservoir in South Brazil. Biota Neotrop. May/Aug 2007 vol. 7, no. 2 http://www.biotaneotropica.org.br/v7n2/pt/abstract?article+bn00807022007. ISSN 1676-0603.

The Capivara Reservoir is located at the Medium Paranapanema river, between the states of Paraná and São Paulo and was formed in 1977. Samples made in 2001 and 2002 yielded 62 fish species, 31 of which occurred in the studied stretch. The nine most abundant species were chosen for the ecomorphological analysis. Eight ecomorphological attributes related to habitat use and foraging were employed in order to identify existing ecomorphological patterns among the species as well as relating them to the main food item consumed by each one. A cluster analysis based on the alimentary index (IAi) data grouped the species according to the main food items consumed. Principal component analysis related piscivores to large heads, caudal fins and terminal mouth with pungent teeth; benthic-dwelling fishes were related to small, subterminal mouths and reduced or absent dentition; omnivores attributes reflected taxonomic relatedness rather than any ecomorphological adaptation; herbivores were related to small mouths and multicuspidated dentition. The resulting trophic groups relative abundances agreed to the general pattern observed in reservoirs, showing a predominance of piscivores, detritivores and omnivores. Cichla monoculus (Spix \& Agassiz, 1831) and Plagioscion squamosissimus (Heckel, 1840) showed large trophic and ecomorphological overlaps meaning that resource use by both species is probably highly similar. Thus ecomorphology seemed to be a suitable tool for describing habitat preferences as well as the prey capture behavior of piscivore species.
\end{abstract}

Keywords: freshwater fishes, numeric ecology, community ecology, trophic ecology.

\section{Resumo}

Teixeira, I. \& S.T. Bennemann. Ecomorfologia refletindo a dieta dos peixes em um reservatório no sul do Brasil. Biota Neotrop. May/Aug 2007 vol. 7, no. 2 http://www.biotaneotropica.org.br/v7n2/pt/abstract?artic le+bn00807022007. ISSN 1676-0603.

O Reservatório de Capivara está localizado no Médio Paranapanema, entre os estados do Paraná e São Paulo, sendo formado em 1977. Coletas realizadas entre 2001 e 2002 resultaram na captura de 62 espécies, sendo 31 no trecho estudado. Destas, as nove mais abundantes foram utilizadas para análise ecomorfológica. Oito atributos ecomorfológicos relacionados ao uso do habitat e alimentação foram usados com objetivo de distinguir padrões ecomorfológicos entre as espécies e relacioná-los ao principal alimento consumido. Um dendrograma de similaridade alimentar foi construído a partir dos resultados do Índice Alimentar (IAi), agrupando as espécies conforme os principais alimentos consumidos. Pela análise de componentes principais piscívoros foram relacionados a cabeças, nadadeiras caudais e bocas terminais grandes com dentes pungentes; peixes bentófagos foram relacionados a bocas pequenas e subterminais e dentição pouco desenvolvida ou ausente; peixes onívoros revelaram atributos relacionados mais à afinidade taxonômica que a adaptações ecomorfológicas; peixes herbívoros foram relacionados a boca pequena e dentes multicuspidados. Os resultados mostraram que as abundâncias relativas dos grupos tróficos estiveram de acordo com o padrão geral observado em reservatórios, com predomínio de piscívoros, detritívoros e onívoros. Cichla monoculus (Spix \& Agassiz, 1831) e Plagioscion squamosissimus (Heckel, 1840) apresentaram grande semelhança ecomorfológica e trófica entre si, permitindo concluir que o uso dos recursos por ambas as espécies é também bastante semelhante. A ecomorfologia se mostrou uma ferramenta adequada na descrição das preferências de habitat das espécies e de aspectos de comportamento de captura de presas entre os piscívoros.

Palavras-chave: peixes de água doce, ecologia numérica, ecologia de comunidades, ecologia trófica. 


\section{Introdução}

A ecomorfologia tem como idéia fundamental que as formas dos organismos e seus modos de vida estão correlacionados, sendo que o ambiente age sobre o fenótipo através de pressões seletivas, selecionando organismos de acordo com os recursos disponíveis no ambiente (Norton et al. 1995). Variações morfológicas entre as espécies refletiriam, ao menos em parte, o uso diferenciado de recursos e por fim, ecologias diferentes, havendo um paralelo entre similaridade morfológica e ecológica (Pianka 2000). Acredita-se que um dado conjunto de caracteres morfológicos indicaria um ajuste a uma dada dimensão do nicho ecológico, possibilitando até certo ponto que previsões acerca da distribuição das espécies pelo ambiente (Watson \& Balon 1984, Barrella et al. 1994, Beaumord \& Petrere-Jr. 1994) ou delimitação de grupos tróficos (Hugueny \& Pouilly 1999, Pouilly et al. 2003) sejam feitas. Assim, análises ecomorfológicas aparecem como ferramentas interessantes no estudo de estrutura de comunidades. No Brasil, os primeiros estudos ecomorfológicos datam do início dos anos 90, com um incremento em meados da década passada, sendo freqüentemente aplicados à fauna marinha e de água doce (Balon et al. 1986, Winemiller et al. 1995, Clifton \& Motta 1998) e apenas um contempla peixes de reservatório (Freire \& Agostinho 2001).

Reservatórios são formados pelo barramento de porções de rios, causando mudanças drásticas ao ambiente (Araújo-Lima et al. 1995, Lowe-McConnell 1999, Mérona et al. 2001). Como conseqüência destas alterações, a comunidade de peixes e de outros organismos sofre rearranjos, com a colonização bem sucedida do ambiente por determinadas espécies e diminuição, ou mesmo perda, de outras (Agostinho et al. 1999).

É esperado para as próximas décadas um aumento no número de reservatórios construídos para fins de aproveitamento do potencial hidroelétrico dos rios brasileiros, com novas usinas sendo construídas na região Sul, Sudeste e principalmente no Centro-Oeste e Norte (MME/CCPE 2002). Assim, estes ambientes serão elementos cada vez mais comuns nas bacias hidrográficas existentes no país. Ao contrário do esperado aumento da área represada das bacias, pouco se conhece sobre o uso do espaço morfológico e sua relação com a dieta de espécies de peixes, em especial onde se registra a ocorrência de espécies alóctones. Tal fato adquire especial importância se considerarmos que reservatórios são ambientes peculiares, não se enquadrando como ambientes lacustres nem fluviais e cujos aspectos estruturais e funcionais de suas comunidades ainda estão por ser adequadamente elucidados (Agostinho et al. 1999).

Ao longo do rio Paranapanema, um afluente da margem esquerda do Alto Paraná, existem hoje 10 usinas hidroelétricas em operação (Duke Energy 2003), entre elas, a UHE Escola Mackenzie, cujo reservatório (Capivara) foi completamente formado em 1977. Recentemente, em quatro trechos diferentes do reservatório (Cinzas, Cruzália, Sertanópolis e Porecatu), foram registradas 62 espécies de peixes (Hoffmann et al. 2005), sendo que o trecho de Porecatu, contendo 31 espécies, é o que melhor caracteriza, em termos gerais, o ambiente de reservatório, devido ao seu caráter semi-lótico. Segundo o exposto acima e de acordo com a teoria ecomorfológica, espera-se que a morfologia reflita adaptações dos peixes existentes no reservatório, com a estrutura da assembléia refletindo as interrelações ecomorfológicas interespecíficas. O objetivo do presente estudo foi, por meio de uma análise ecomorfológica, distinguir e caracterizar grupos morfológicos entre espécies de peixe de reservatórios, verificando se a ecomorfologia é uma metodologia adequada para indicar preferências alimentares.

\section{Material e métodos}

\section{Caracterização da área de estudos}

O rio Paranapanema corre ao longo de $660 \mathrm{~km}$ de extensão, dos quais $329 \mathrm{~km}$ formam a divisa natural entre os estados do Paraná e de São Paulo (Maack 1981). O reservatório Capivara, formado por um represamento próximo à foz do rio Vermelho, possui $100 \mathrm{~km}$ de comprimento e largura variando de 0,8 a $10 \mathrm{~km}$. A profundidade varia de $70 \mathrm{~m}$, próximo à barragem da UHE Escola Mackenzie, até $6 \mathrm{~m}$ à montante, próximo à barragem da UHE Canoas I. O trecho estudado, localizado no município de Porecatu (Figura 1), é considerado semi-lótico profundo (15 a $70 \mathrm{~m}$ ) e com elevada transparência da água (aproximadamente $2 \mathrm{~m}$ ). O leito do rio é inteiramente rochoso, inserido em uma região de relevo acidentado, encaixado em um vale com morros. Ocorrem afloramentos basálticos em diversos braços. A vegetação nativa é escassa; no entanto, grande quantidade de macrófitas aquáticas foi registrada nas áreas litorâneas.

\section{Amostragens e coleta de dados morfométricos}

Os exemplares de peixes utilizados neste estudo foram provenientes das coletas realizadas por (Hoffmann et al. 2005), no período maio de 2001 e maio de 2002 e mais duas de caráter complementar, a primeira em dezembro de 2003 e a segunda entre junho e julho de 2004. As coletas foram realizadas com redes de espera (malhas 7, 10 e 11) dispostas perpendicularmente às margens da represa, além de tarrafas para coleta de espécies menores nas margens. Varas de pesca com iscas artificiais foram utilizadas para captura de Cichla monoculus nas coletas complementares.

Foram selecionadas as nove espécies principais em termos de abundância (Tabela 1) ao longo do período de amostragem, entre maio de 2001 e maio de 2002. Somente indivíduos adultos, de tamanhos acima da $\mathrm{L}_{50}$ (comprimento médio da primeira maturação gonadal, segundo dados de Vazzoler 1996), foram selecionados para a caracterização morfométrica. Na tomada de dados morfométricos, procurou-se utilizar 10 indivíduos adultos de cada espécie com a menor variação possível de comprimentos padrões; caso contrário utilizou-se o número máximo de indivíduos adultos cujos compri-
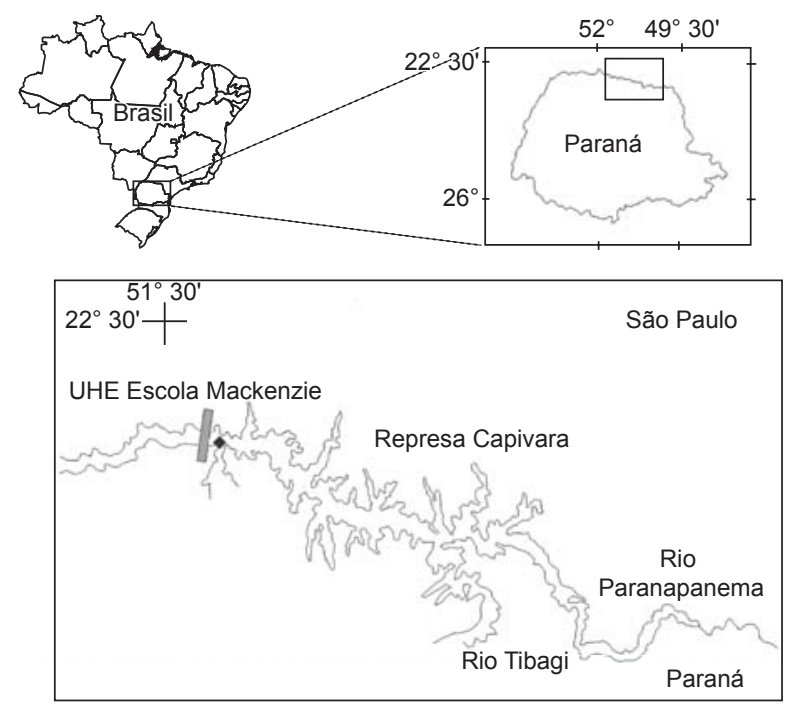

Figura 1. Mapa esquemático mostrando o reservatório Capivara e a UHE Escola Mackenzie. A área de estudos se encontra próxima à barragem.

Figure 1. Schematic map showing the Capivara reservoir and the Escola Mackenzie Power Plant. $\downarrow$ The study área is near the dam. 
mentos padrões fossem mais homogêneos entre si (Tabela 1). Os peixes foram fixados em formalina $10 \%$ e para manter a boca aberta foram utilizados pedaços de madeira ou de papelão para possibilitar posterior mensuração das medidas da boca. Posteriormente, os espécimes foram conservados em álcool $70 \%$. Todos os espécimes utilizados estão depositados no Museu de Zoologia da Universidade Estadual de Londrina (MZUEL).

As medidas morfométricas foram tomadas de acordo com estudos anteriores (Gatz 1979a, 1979b, Watson \& Balon 1984, Balon et al. 1986, Wikramanayake 1990), sendo escolhidas aquelas que refletissem aspectos relacionados ao uso do habitat e alimentação. As medidas absolutas (Tabela 2) foram tomadas em linha reta entre pontos, através de paquímetro digital (precisão de $0,01 \mathrm{~mm}$ ) para medidas até $120 \mathrm{~mm}$ e ictiômetro (precisão de $1 \mathrm{~mm}$ ), para medidas superiores a $120 \mathrm{~mm}$. A partir destas medidas, foram calculados os atributos ecomorfológicos (Tabela 3). As áreas do corpo e das nadadeiras foram calculadas baseando-se em metodologia utilizada por Wikramanayake (1990). Utilizou-se para isso uma placa de isopor sobre a qual se posicionou uma folha de papel milimetrado. $\mathrm{O}$ peixe foi posto esticado sobre uma folha de acetato interposta entre o peixe e o papel milimetrado de forma a não molhá-lo. alfinetes de metal foram utilizados na realização do contorno do corpo e das nadadeiras, devidamente esticadas, e os furos produzidos deste modo foram ligados posteriormente com uma lapiseira. As figuras formadas foram recortadas e coladas em folhas de papel branco, posteriormente fotocopiadas em tamanho original, utilizando-se papel sulfite A4 de gramatura constante. As figuras fotocopiadas foram recortadas e pesadas em balança analítica. Por fim, seus pesos foram comparados com o de folhas A4 da mesma gramatura, para que a área pudesse ser estimada a partir do peso através de regressão linear. A mesma metodologia foi aplicada para a obtenção da área das nadadeiras peitorais.

\section{Análises}

Além dos atributos ecomorfológicos medidos e utilizados nas análises estatísticas, foram observadas também a dentição e a orientação da boca dos peixes. As medidas relativas das espécies foram submetidas à análise de componentes principais ( $\mathrm{ACP})$, feita através do programa PaST versão 1.32 (Hammer et al. 2004), utilizando-se uma matriz de correlação, tendo em vista as unidades diferentes das medidas utilizadas. Por meio desta análise de ordenação de dados multivariados, um grande conjunto de variáveis é sintetizado em um

Tabela 1. Relação das espécies de maior abundância coletadas no reservatório Capivara, contendo o número de indivíduos e sua porcentagem em relação ao total coletado no trecho Porecatu, no período de maio/2001 a maio/2002. Fonte: Hoffmann et al. (2005).

Table 1. List of the most abundant species captured in the Capivara Reservoir containing the number of individuals, and their percentage in relation to the total catch in the study area, May/2001 to May/2002. Source: Hoffmann et al. (2005).

\begin{tabular}{|c|c|c|c|c|}
\hline Espécies & Acrônimo & $\begin{array}{c}\mathrm{n}^{0} \text { de indivíduos } \\
\text { medidos }\end{array}$ & $\begin{array}{c}\mathrm{n}^{0} \text { de indivíduos } \\
\text { coletados }\end{array}$ & $\begin{array}{c}\% \text { de indivíduos } \\
\text { coletados }\end{array}$ \\
\hline Astyanax altiparanae & Aalt & 9 & 226 & 23,54 \\
\hline Cichla monoculus & Cmon & 5 & 181 & 18,85 \\
\hline Apareiodon affinis & Aaff & 10 & 130 & 13,54 \\
\hline Plagioscion squamosissimus & Psqu & 9 & 70 & 7,29 \\
\hline Steindachnerina insculpta & Sins & 10 & 51 & 5,31 \\
\hline Schizodon nasutus & Snas & 10 & 45 & 4,69 \\
\hline Acestrorhynchus lacustris & Alac & 9 & 36 & 3,75 \\
\hline Moenkhausia intermedia & Mint & 5 & 34 & 3,54 \\
\hline Iheringichthys labrosus & Ilab & 10 & 26 & 2,71 \\
\hline Soma dos indivíduos das 9 espécies & & & 773 & 83,22 \\
\hline Total de indivíduos coletados das 31 espécies & & & 960 & 100 \\
\hline
\end{tabular}

Tabela 2. Medidas absolutas utilizadas nas análises morfométricas.

Table 2. Absolute measurements used on the morphometric analyses.

\begin{tabular}{|c|c|c|}
\hline Medida & Sigla & Explicação \\
\hline Comprimento padrão & $\mathrm{CP}$ & distância entre a ponta do focinho e a extremidade da última vértebra caudal \\
\hline Comprimento da cabeça & $\mathrm{CC}$ & distância entre a ponta do focinho e a extremidade posterior do opérculo \\
\hline Altura da linha do olho & ALO & $\begin{array}{l}\text { altura tomada da borda ventral da cabeça ao meio do olho, na mesma linha da altura da } \\
\text { cabeça }\end{array}$ \\
\hline Altura da cabeça & $\mathrm{AC}$ & distância entre a borda ventral à dorsal da cabeça na mesma linha da altura do olho \\
\hline Comprimento do dentário & $\mathrm{CD}$ & distância entre a extremidade anterior do dentário à articulação com o suspensório \\
\hline Altura máxima do corpo & AMC & máxima distância entre a borda ventral e dorsal do corpo (exceto nadadeiras) \\
\hline Largura máxima do corpo & LMC & máxima distância transversal do corpo \\
\hline Comprimento do pedúnculo & $\mathrm{CPe}$ & $\begin{array}{l}\text { distância entre a borda posterior da base da nadadeira anal à borda posterior da última } \\
\text { vértebra }\end{array}$ \\
\hline Altura do pedúnculo & $\mathrm{APe}$ & altura do pedúnculo tomada na região de menor altura \\
\hline Largura do pedúnculo & $\mathrm{LPe}$ & distância transversal tomada na mesma região da altura do pedúnculo \\
\hline
\end{tabular}


Tabela 3. Atributos utilizados nas análises ecomorfológicas.

Table 3. Attributes used on the ecomorphological analyses.

\begin{tabular}{|c|c|c|}
\hline Atributo & Fórmula & Interpretação \\
\hline $\begin{array}{l}\text { Comprimento relativo da cabeça } \\
\text { (CRC) }\end{array}$ & $\mathrm{CC} / \mathrm{CP}$ & $\begin{array}{l}\text { Atributo diretamente relacionado ao tamanho relativo do alimento } \\
\text { consumido (Gatz 1979b) }\end{array}$ \\
\hline Altura relativa do olho (ARO) & $\mathrm{ALO} / \mathrm{AC}$ & $\begin{array}{l}\text { Altos valores indicam olhos dorsalmente posicionados, característico } \\
\text { de peixes bentônicos (Watson \& Balon 1984) }\end{array}$ \\
\hline $\begin{array}{l}\text { Índice de compressão do corpo } \\
\text { (ICC) }\end{array}$ & AMC/LMC & $\begin{array}{l}\text { Valores elevados indicam peixe lateralmente comprimido, relacionan- } \\
\text { do-se a peixes que ocupam habitats de águas lentas (Watson \& Balon, } \\
\text { 1984), podendo indicar também preferência por águas superficiais } \\
\text { (Pouilly et al. 2003) }\end{array}$ \\
\hline Altura relativa do corpo (ARC) & $\mathrm{AMC} / \mathrm{CP}$ & $\begin{array}{l}\text { Atributo inversamente relacionado com ambientes de hidrodinamismo } \\
\text { elevado e diretamente relacionado com a capacidade de desenvolver } \\
\text { deslocamentos verticais (Gatz 1979b) }\end{array}$ \\
\hline $\begin{array}{l}\text { Comprimento relativo do pedún- } \\
\text { culo }(\mathrm{CRPe})\end{array}$ & $\mathrm{CPe} / \mathrm{CP}$ & $\begin{array}{l}\text { Pedúnculos longos indicam bons nadadores, inclusive peixes bentô- } \\
\text { nicos habitantes em ambientes de hidrodinamismo elevado (Watson } \\
\text { \& Balon 1984) }\end{array}$ \\
\hline $\begin{array}{l}\text { Índice de compressão do pedún- } \\
\text { culo (ICPe) }\end{array}$ & $\mathrm{APe} / \mathrm{LPe}$ & $\begin{array}{l}\text { Pedúnculos comprimidos indicam indivíduos de natação lenta e pouca } \\
\text { manobrabilidade, podendo afetar o desempenho em arrancadas rápidas } \\
\text { à medida que aumenta a altura dos corpos entre as diferentes espécies } \\
\text { (Gatz 1979b) }\end{array}$ \\
\hline $\begin{array}{l}\text { Área relativa da nadadeira caudal } \\
\text { (ArRNC) }\end{array}$ & $\begin{array}{l}\text { área da nadadeira caudal/ } \\
\text { área do corpo }\end{array}$ & $\begin{array}{l}\text { Grandes nadadeiras caudais indicam movimentos em arrancadas } \\
\text { rápidas, modo típico de natação de vários peixes bentônicos (Balon } \\
\text { et al. 1986) }\end{array}$ \\
\hline $\begin{array}{l}\text { Área relativa da nadeira peitoral } \\
\text { (ArRNPet) }\end{array}$ & $\begin{array}{l}\text { área da nadadeira peitoral/ } \\
\text { área do corpo }\end{array}$ & $\begin{array}{l}\text { Valores altos são encontrados em nadadores lentos, que usam a na- } \\
\text { dadeira para manobras e frenagens, ou habitantes de águas correntes } \\
\text { que as usam como defletores de corrente, possibilitando dessa maneira } \\
\text { manterem-se em contato com o substrato (Gatz 1979b) }\end{array}$ \\
\hline $\begin{array}{l}\text { Abertura relativa da boca } \\
(\mathrm{ARBo})\end{array}$ & $\mathrm{CD} / \mathrm{SL}$ & $\begin{array}{l}\text { Altos valores indicam grande abertura da boca, indicando alimentação } \\
\text { baseada em itens de porte relativamente grandes }\end{array}$ \\
\hline
\end{tabular}

menor número de eixos que o original através de novas correlações lineares estabelecidas entre as variáveis. Adotou-se por convenção a análise dos componentes cujos autovalores sejam maiores que 1 , pois a partir de valores menores a influência da variância residual é cada vez maior, dificultando a análise. Para definição dos grupos ecomorfológicos, considerou-se em conjunto a que grupos tróficos pertencem as espécies e os atributos ecomorfológicos em comum, com a abundância relativa de cada grupo ecomorfológico sendo a soma percentual das espécies capturadas no período 2001/2002.

A alimentação das espécies de peixes foi analisada pela frequiência de ocorrência e peso dos itens alimentares identificados (Hyslop 1980) e interpretados por meio do índice alimentar (IAi), substituindo o percentual em volume pelo percentual em peso (Kawakami \& Vazzoler 1980, modificado), dado pela equação:

$$
\mathrm{IAi}=\% \mathrm{Fi} \times \% \mathrm{Pi} / \Sigma(\% \mathrm{Fi} \times \% \mathrm{Pi})
$$

onde: $\mathrm{IAi}=$ Índice Alimentar; $\% \mathrm{Fi}=$ Percentual da frequiência de ocorrência do item alimentar na espécie $\mathrm{i}$; e \% $\mathrm{Pi}=$ Percentual do peso da categoria alimentar na dieta da espécie i;

Os valores do IAi foram utilizados para determinar a similaridade trófica entre as espécies, utilizando o índice quantitativo de BrayCurtis e posterior agrupamento pelo método de ligações completas (Valentin 2000). Tais análises foram processadas com auxílio do programa PaST versão 1.32 (Hammer et al. 2004). Para o cálculo das correlações entre morfologia e dieta das espécies de peixes, foi utilizado o teste de Mantel, realizado através do programa XLStat (Kovach Computing Services 2004). Para isso, duas matrizes triangulares, uma contendo as distâncias morfológicas e outra as distâncias tróficas entre as espécies, foram elaboradas, a primeira por meio do cálculo das distâncias euclidianas e a segunda, por meio do índice quantitativo de Bray-Curtis, baseado nos valores obtidos pelo IAi.

\section{Resultados}

\section{Análise de componentes principais}

A análise de componentes principais (ACP) produziu três eixos com autovalores maiores que 1,0 (Tabela 4).

Os atributos que mais contribuíram para a variância do primeiro eixo (CP1) foram ArRNPet, CRC, ArRNC apresentando valores positivos, e ARC, ICPe e ICC, com valores negativos. Astyanax altiparanae e Moenkhausia intermedia foram caracterizados como tendo corpos lateralmente comprimidos e altos e cabeça relativamente pequena; Acestrorhynchus lacustris, Iheringichthys labrosus, Apareiodon affinis, Cichla monoculus e Plagioscion squamosissimus, caracterizados como tendo nadadeiras caudais e peitorais grandes, corpos relativamente baixos, de secção transversal larga, pedúnculo caudal relativamente longo e cabeças relativamente grandes; Schizodon nasutus e Steindachnerina insculpta estão em posição intermediária no eixo, entre peixes de corpos lateralmente comprimidos e altos e peixes de corpos mais largos e relativamente baixos, podendo ser considerados como detentores de corpos fusiformes, além de possuírem nadadeiras caudais e peitorais, além da cabeça, com proporções intermediárias.

$\mathrm{O}$ segundo eixo (CP2) teve como principais contribuições à sua variância os atributos $\mathrm{CRC}, \mathrm{ICC}, \mathrm{ARC}$ e ARBo apresentando valores negativos. Plagioscion squamosissimus, $C$. monoculus e $A$. altiparanae foram caracterizados como tendo cabeças e bocas relati- 
vamente grandes, além de corpos caracterizados como relativamente altos possuindo compressão lateral. Schizodon nasutus e A. affinis apresentaram escores positivos projetados em $\mathrm{CP} 2$, sendo caracterizadas como espécies de cabeças e bocas relativamente pequenas e de corpos baixos de secção transversal mais larga. Steindachnerina insculpta, A. lacustris, M. intermedia e I. labrosus situaram-se em posições intermediárias ao longo de $\mathrm{CP} 2$, com os corpos tendendo a um formato mais fusiforme (Figura 2).

O terceiro eixo (CP3) teve como principal atributo em sua formação ARBo, apresentando valor positivo, e ARO, ICPe e CRPe, com valores negativos. Plagioscion squamosissimus, I. labrosus e A. affinis apresentaram em geral escores negativos ao longo de CP3 sendo caracterizados como espécies detentoras de olhos tendendo a uma posição dorsal, pedúnculos caudais longos e espessos. Moenkhausia intermedia e A. altiparanae apresentaram projeções próximas a valores intermediários e negativos ao longo de CP3, a última tendendo a valores mais negativos. Em conjunto apresentaram influência de atributos que as caracterizam como peixes de bocas relativamente pequenas, olhos laterais, com pedúnculos caudais curtos e comprimidos. Steindachnerina insculpta e principalmente $C$. monoculus e S. nasutus, tiveram seus escores projetados em posição intermediária
Tabela 4. Resultados da análise de componentes principais nos três primeiros eixos calculados para as espécies de peixes do Reservatório Capivara. Valores em negrito destacam os escores que mais contribuíram para a variância observada.

Table 4. Principal component analysis results for the first three axis calculated for the Capivara Reservoir fish species. Values in bold highlight the most contributing scores to the observed variance.

\begin{tabular}{lccc}
\hline \multicolumn{1}{c}{ Atributos } & CP1 (3,502) & CP2 (1,898) & CP3 (1,528) \\
\hline CRC & 0,345 & $-0,472$ & 0,171 \\
ARO & 0,287 & $-0,051$ & $-0,479$ \\
ICC & $-0,325$ & $-0,473$ & $-0,004$ \\
ARC & $-0,359$ & $-0,383$ & $-0,227$ \\
CRPe & 0,291 & $-0,192$ & $-0,535$ \\
ICPe & $-0,348$ & 0,037 & $-0,441$ \\
ArRNC & 0,335 & 0,157 & 0,215 \\
ArRNPet & 0,431 & $-0,055$ & $-0,283$ \\
ArRBo & 0,166 & $-0,582$ & 0,289 \\
Variância (\%) & 40,6 & 22,0 & 17,7 \\
Variância total & & & 80,3 \\
\hline
\end{tabular}

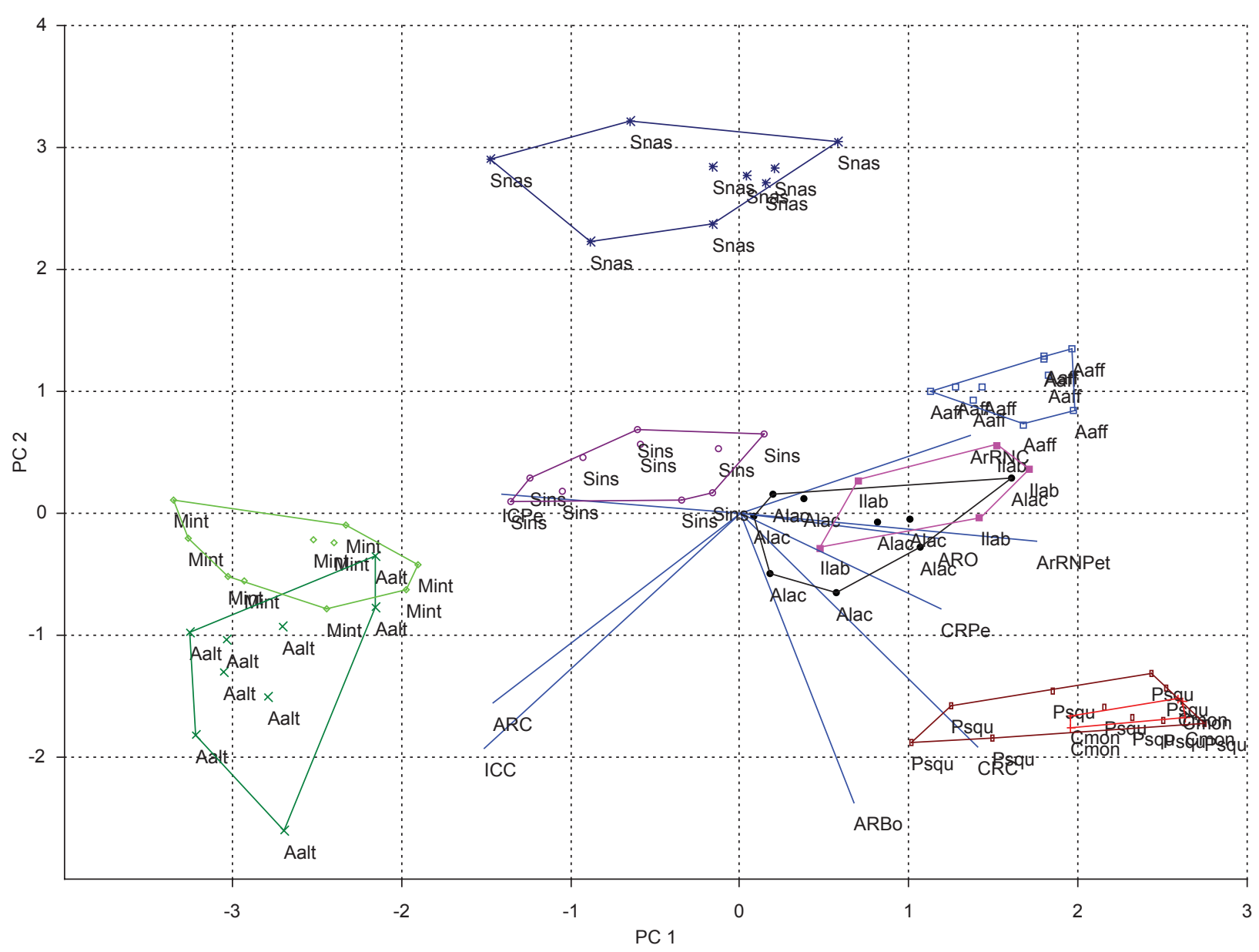

Figura 2. Projeções nos escores nos eixos 1 e 2 da análise de componentes principais com os atributos das nove espécies estudadas no reservatório Capivara. $($ Alac $)=$ Acestrorhynchus lacustris $;($ Cmon $)=$ Cichla monoculus $;($ Aaff $)=$ Apareiodon affinis $;($ Aalt $)=$ Astyanax altiparanae $;($ Sins $)=$ Steindachnerina insculpta $;$ $($ Mint $)=$ Moenkhausia intermedia $;($ Snas $)=$ Schizodon nasutus $;($ Ilab $)=$ Iheringichthys labrosus $;($ Psqu $)=$ Plagioscion squamosissimus .

Figure 2. Projections of the first two principal components with the attributes of the 9 species studied in the Capivara reservoir. (Alac) = Acestrorhynchus lacustris $;($ Cmon $)=$ Cichla monoculus $;($ Aaff $)=$ Apareiodon affinis $;($ Aalt $)=$ Astyanax altiparanae $;($ Sins $)=$ Steindachnerina insculpta $;($ Mint $)=$ Moenkhausia intermedia $;($ Snas $)=$ Schizodon nasutus $;(\mathrm{Ilab})=$ Iheringichthys labrosus $;(\mathrm{Psqu})=$ Plagioscion squamosissimus . 
ao longo de $\mathrm{CP} 3$, ainda sugerindo formas intermediárias entre corpos altos e comprimidos e corpos relativamente mais baixos e robustos e olhos laterais. Acestrorhynchus lacustris apresentou os escores mais altos em $\mathrm{CP} 3$, sendo por isso caracterizada como espécie de grande abertura bucal, olhos laterais, pedúnculos caudais curtos e espessos (Figura 3).

Além dos atributos analisados nas ACP, foram observadas a orientação da boca e a dentição. Moenkhausia intermedia, A. altiparanae, C. monoculus, P. squamosissimus e A. lacustris apresentaram bocas terminais; A. affinis, I. labrosus, S. insculpta e $S$. nasutus, bocas subterminais. Quanto à dentição, M. intermedia, A. altiparanae e $S$. nasutus apresentaram dentes multicuspidados, aptos a cortar e/ou agarrar; A. lacustris apresentou na boca de formato semelhante a um forceps uma mistura de dentes cônicos e caninos; P. squamosissimus apresentou boca protrátil e dentes cônicos; $C$. monoculus, boca protrátil e numerosos minúsculos dentes cônicos; I. labrosus apresentou boca tubular contendo pequenos dentes viliformes; A. affinis, uma frágil boca de mandíbulas formando uma pá, cujos dentes, incisivos, encontram-se somente no pré-maxilar, sendo aptos à raspagem; $S$. insculpta apresenta boca frágil e sem dentes.

\section{Análise de similaridade alimentar}

A análise de similaridade alimentar, baseada no IAi, (Tabela 5 e Figura 4), separou as espécies carnívoras das demais (insetívoras, detritívoras e herbívoras).

Classificadas como carnívoras estão P. squamosissimus, C. monoculus e A. lacustris, divididas em dois subgrupos: (Psqu + Cmon) e (Alac). Plagioscion squamosissimus e C. monoculus tiveram mais de $70 \%$ de similaridade na dieta, sendo o grupo formado baseado em uma alimentação composta de peixes e camarões. Tanto $P$. squamosissimus quanto $C$. monoculus tiveram a dieta composta em aproximadamente $50 \%$ por camarões. Entre os peixes predados por C. monoculus estão principalmente juvenis da própria espécie e restos de peixes não identificados, além de cascudos (Loricariidae), Oreochromis niloticus e juvenis de P. squamosissimus em menor quantidade. Plagioscion squamosissimus apresentou em sua dieta uma grande quantidade de peixes não identificáveis nas análises de conteúdo estomacal, além de espécimes juvenis de C. monoculus, Gymnotus carapo e juvenis da própria espécie. Já A. lacustris, que apresentou similaridade de quase $50 \%$ na dieta em relação ao primeiro

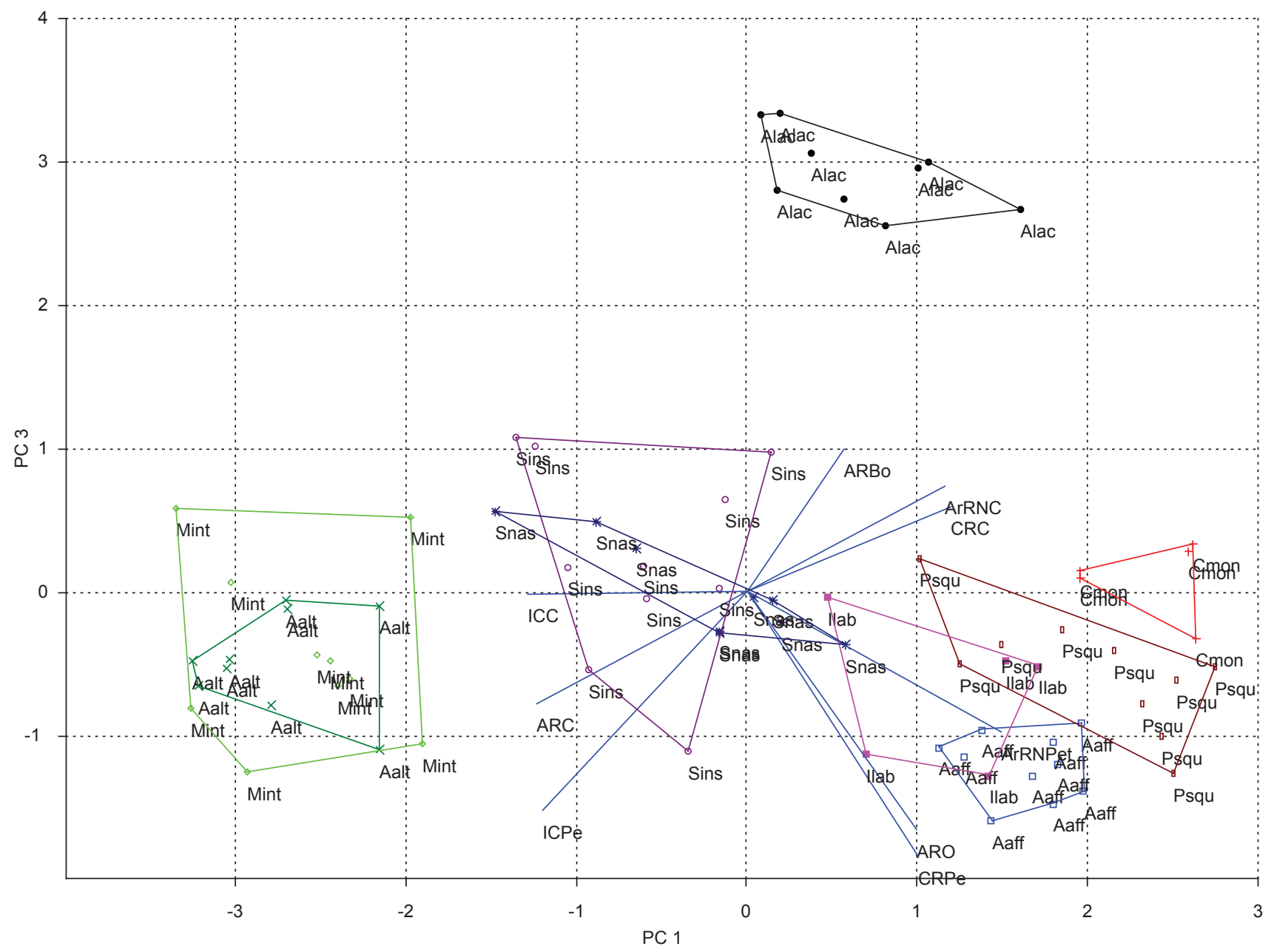

Figura 3. Projeções nos escores nos eixos 1 e 3 da análise de componentes principais com os atributos das nove espécies estudadas no reservatório Capivara. $($ Alac $)=$ Acestrorhynchus lacustris $;($ Cmon $)=$ Cichla monoculus $;($ Aaff $)=$ Apareiodon affinis $;($ Aalt $)=$ Astyanax altiparanae $;($ Sins $)=$ Steindachnerina insculpta $;$ $($ Mint $)=$ Moenkhausia intermedia $;($ Snas $)=$ Schizodon nasutus $;(\mathrm{Ilab})=$ Iheringichthys labrosus $;(\mathrm{Psqu})=$ Plagioscion squamosissimus.

Figure 3. Projections of the first and third principal components with the attributes of the 9 species studied in the Capivara reservoir. (Alac) $=$ Acestrorhynchus lacustris $;(\mathrm{Cmon})=$ Cichla monoculus; $($ Aaff $)=$ Apareiodon affinis; $($ Aalt $)=$ Astyanax altiparanae $;($ Sins $)=$ Steindachnerina insculpta $;($ Mint $)=$ Moenkhausia intermedia $;($ Snas $)=$ Schizodon nasutus $;($ Ilab $)=$ Iheringichthys labrosus $;($ Psqu $)=$ Plagioscion squamosissimus. 
Tabela 5. Índice alimentar (IAi) das espécies de peixes estudadas no reservatório de Capivara. Os valores sombreados indicam componentes importantes na dieta. Cichla monoculus (Cmon), Plagioscion squamosissimus (Psqu), Acestrorhynchus lacustris (Alac), Iheringichthys labrosus (Ilab), Apareiodon affinis (Aaff), Steindachnerina insculpta (Sins), Moenkhausia intermedia (Mint), Schizodon nasutus (Snas) e Astyanax altiparanae (Aalt).

Table 5. Alimentary Index (IAi) of the fish species studied in the Capivara Reservoir. Shaded values indicate important diet components. Cichla monoculus (Cmon), Plagioscion squamosissimus (Psqu), Acestrorhynchus lacustris (Alac), Iheringichthys labrosus (Ilab), Apareiodon affinis (Aaff), Steindachnerina insculpta (Sins), Moenkhausia intermedia (Mint), Schizodon nasutus (Snas) e Astyanax altiparanae (Aalt).

\begin{tabular}{|c|c|c|c|c|c|c|c|c|c|}
\hline Itens & Cmon & Psqu & Alac & Ilab & Aaff & Sins & Mint & Snas & Aalt \\
\hline \multicolumn{10}{|l|}{ Vegetais } \\
\hline Gramínea (folhas) & - & - & - & - & - & - & - & 39,2 & - \\
\hline Gramínea (sementes) & - & - & - & - & - & - & - & & 3,3 \\
\hline Algas filamentosas & - & - & - & - & 17,1 & - & 0,1 & 4,8 & 3,2 \\
\hline Quirela & - & - & - & - & & - & - & & 13,4 \\
\hline Restos vegetais & - & 1,9 & - & 4,3 & 0,6 & - & 18,3 & 34,3 & 17,9 \\
\hline \multicolumn{10}{|l|}{ Insetos } \\
\hline Ceratopogonidae & - & - & - & 0,3 & - & - & - & - & - \\
\hline Chaoboridae & - & - & - & 0,4 & - & - & - & - & - \\
\hline Chironomidae & 0,1 & - & - & 69,2 & - & - & - & - & 0,6 \\
\hline Coleoptera & - & - & - & - & - & - & - & - & 0,4 \\
\hline Diptera (outros) & - & - & - & 0,1 & - & - & - & - & 2,2 \\
\hline Ephemeroptera & - & 0,1 & - & 0,5 & - & - & - & - & 10,2 \\
\hline Hymenoptera & 0,1 & - & - & - & - & - & - & - & 0,4 \\
\hline Odonata & - & 2,2 & - & - & - & - & 0,7 & - & 2,2 \\
\hline Trichoptera & - & - & - & 0,2 & - & - & - & - & - \\
\hline Insetos (restos) & 0,8 & 0,1 & - & 1,0 & - & - & - & - & 23,4 \\
\hline \multicolumn{10}{|l|}{ Crustáceos } \\
\hline Camarão & 53,7 & 56,7 & - & - & - & - & - & - & 1,2 \\
\hline Cladocera & 0,4 & - & - & 0,1 & - & - & - & - & 8,0 \\
\hline Copepoda & 4,1 & - & - & 2,2 & - & - & 2,6 & - & 0,4 \\
\hline Ostracoda & - & - & - & 2,2 & - & - & 11,6 & - & - \\
\hline \multicolumn{10}{|l|}{ Peixes } \\
\hline A. altiparanae & - & 0,7 & - & - & - & - & - & - & - \\
\hline A. affinis & - & - & 4,3 & - & - & - & - & - & - \\
\hline C. monoculus & 26,8 & 7.1 & 7,7 & - & - & - & - & - & - \\
\hline G. carapo & - & 6,6 & - & - & - & - & - & - & - \\
\hline Loricariidae & 0,1 & - & - & - & - & - & - & - & - \\
\hline O. niloticus & 0,3 & - & - & - & - & - & - & - & - \\
\hline P. squamosissimus & 0,3 & 2,7 & 1,5 & - & - & - & - & - & - \\
\hline Peixes (restos) & 13,2 & 22,1 & 86,6 & - & - & - & - & - & 2,7 \\
\hline \multicolumn{10}{|l|}{ Outros itens } \\
\hline Bivalvia & - & - & - & 1,1 & - & - & - & - & - \\
\hline Gastropoda & - & - & - & 0,4 & - & - & - & - & 4,2 \\
\hline Detrito & - & - & - & 17,4 & 71,7 & 40,3 & 66,7 & 20,7 & 4,8 \\
\hline Sedimento & - & - & - & 2,9 & 10,6 & 59,5 & & 1,0 & 1,5 \\
\hline
\end{tabular}

grupo (Psqu + Cmon), alimentou-se exclusivamente de peixes, apresentando grande parte de presas não identificáveis. Dentre as espécies identificadas estão C. monoculus (juvenis), A. affinis e juvenis de P. squamosissimus.

O agrupamento contendo as demais espécies é formado por A. affinis, S. insculpta, M. intermedia, S. nasutus, I. labrosus e A. altiparanae. A última apresenta uma baixa similaridade em sua dieta em relação às demais espécies do agrupamento devido à grande variedade de itens alimentares consumidos, principalmente insetos e alimentos de origem vegetal, sendo por isso uma espécie onívora. Ao contrário das outras espécies do grupo, A. altiparanae foi a única a consumir quirela (ceva), sendo inclusive parte importante da dieta, reforçando sua classificação como oportunista. Ademais, foi a única, em conjunto com I. labrosus, a conter uma grande variedade de insetos em sua dieta, apresentando basicamente restos de insetos e náiades de Ephemeroptera. Iheringichthys labrosus separa-se do grupo formado por (Aaff + Sins + Mint + Snas) devido à sua dieta constituída basicamente de larvas de Chironomidae e em menor importância, detrito e sedimento. O restante do grupo é subdividido em dois subgrupos, (Aaff + Sins) e (Mint + Snas). Apareidon affinis e S. insculpta foram agrupados devido ao alto consumo de detrito e sedimento apresentado por ambas as espécies. Porém, ao contrário de $S$. insculpta, A. affinis 
consumiu quantidade expressiva de algas filamentosas, enquanto que $S$. insculpta teve sua dieta composta exclusivamente de detrito, ingerido em conjunto com sedimento. Schizodon nasutus e $M$. intermedia foram agrupadas devido ao consumo de matéria vegetal e detrito. Entretanto, $S$. nasutus apresentou uma dieta constituída em grande parte por restos vegetais (p.ex., folhas de gramínea), enquanto $M$. intermedia consumiu principalmente detrito e em menor quantidade, restos vegetais, Ostracoda e Copepoda.

A dieta, morfologia externa e dentição, quando analisadas em conjunto, permitem agrupar as espécies em três agrupamentos ecomorfológicos. A contribuição em percentagem de abundância de cada espécie entre as estudadas (conforme Tabela 1), assim como

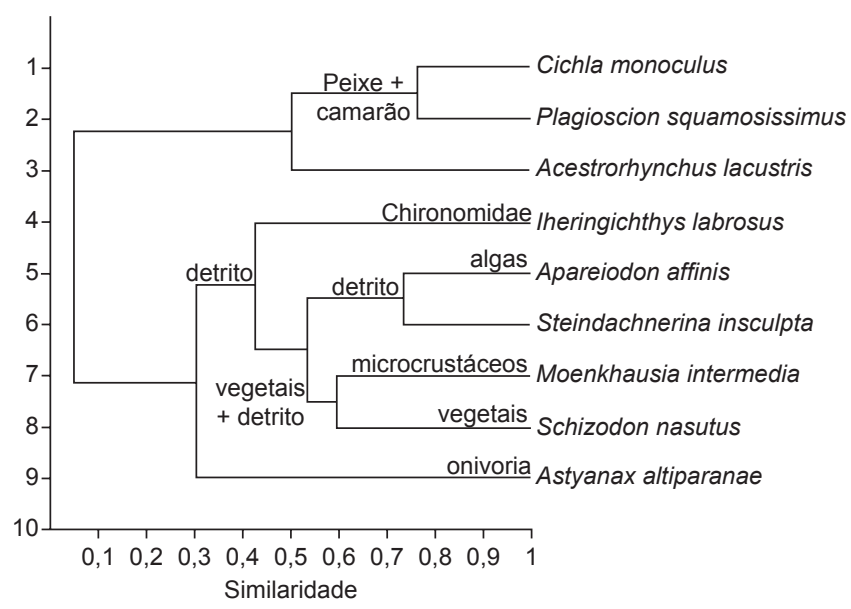

Figura 4. Dendrograma de similaridade alimentar a partir dos resultados dos valores do Índice Alimentar (IAi) das nove espécies de peixes do reservatório Capivara.

Figure 4. Dendrogram of alimentary similarity based on the Alimentary Index (IAi) from the nine fish species of the Capivara reservoir. a contribuição em percentagem de cada padrão ecomorfológico, é dada na Tabela 6.

Os resultados do teste de Mantel aplicado às matrizes de distâncias tróficas e morfológicas mostraram correlação significativa ( $r=0,31 ; P=0,047$, a partir de 362800 permutações) entre os atributos morfológicos e os alimentos consumidos pelas espécies estudadas, apoiando assim a hipótese de existência de relação entre a morfologia dos peixes e suas dietas.

\section{Discussão}

Trabalhos ecomorfológicos realizados nas últimas duas décadas apresentaram resultados contrastantes entre si. Enquanto determinados autores encontraram relações consistentes entre morfologia e ecologia (Mahon 1984, Watson \& Balon 1984, Wikramanayake 1990, Winemiller et al. 1995), outros observaram apenas fraca correlação (Douglas \& Matthews 1992) ou mesmo ausência de relação entre forma e ecologia das espécies (Felley 1984). No presente trabalho, foi possível observar padrões ecomorfológicos entre as espécies, conforme exposto a seguir.

As espécies estudadas no reservatório Capivara foram divididas em quatro grupos tróficos, de acordo com os resultados de similaridade alimentar. Cada um destes grupos apresentou entre seus membros atributos ecomorfológicos em comum, ainda que tenham sido observadas também variações intra-grupo no que concerne à forma do corpo.

As espécies $P$. squamosissimus, C. monoculus e A. lacustris somaram $30 \%$ dos indivíduos coletados, representando os carnívoros piscívoros. Apresentaram em comum cabeças relativamente grandes, nadadeiras caudais de grande área e pedúnculos caudais de secção transversal relativamente larga. Este conjunto de atributos indica peixes que se alimentam de presas grandes, tendo boa capacidade natatória, podendo realizar arranques a partir do repouso. A boca terminal, de grande abertura e portando dentes cônicos ou pontiagudos, relaciona-se nitidamente à dieta baseada em organismos evasivos, com os dentes tornando mais eficiente a apreensão do alimento. Plagioscion

Tabela 6. Percentuais de abundância das nove espécies estudadas do reservatório Capivara, agrupados por padrões ecomorfológicos e dieta. Plagioscion squamosissimus (Psqu), Cichla monoculus (Cmon), Acestrorhynchus lacustris (Alac), Iheringichthys labrosus (Ilab), Apareiodon affinis (Aaff), Steindachnerina insculpta (Sins), Moenkhausia intermedia (Mint), Astyanax altiparanae (Aalt) e Schizodon nasutus (Snas). Acrônimos dos atributos conforme Tabela 3.

Table 6. Relative abundance of the nine species studied in the Capivara Reservoir clustered according to ecomorphological patterns and diet. Plagioscion squamosissimus (Psqu), Cichla monoculus (Cmon), Acestrorhynchus lacustris (Alac), Iheringichthys labrosus (Ilab), Apareiodon affinis (Aaff), Steindachnerina insculpta (Sins), Moenkhausia intermedia (Mint), Astyanax altiparanae (Aalt) e Schizodon nasutus (Snas). Attributes acronyms according to Table 3.

\begin{tabular}{|c|c|c|c|c|}
\hline Espécies & $\begin{array}{l}\text { Indivíduos por } \\
\text { espécie }(\%)\end{array}$ & $\begin{array}{c}\text { Grupo } \\
\text { morfológico }(\%)\end{array}$ & Atributos relacionados & Guilda trófica \\
\hline Psqu & 7,29 & \multirow[t]{3}{*}{29,89} & \multirow{3}{*}{$\begin{array}{l}\text { ARBo, CRC, ArRNC, ArRNPet (positivos); ICC, } \\
\text { ICPe, ARC (negativos); boca terminal, dentes ca- } \\
\text { ninos e/ou cônicos }\end{array}$} & \multirow[t]{3}{*}{ Nectônicos carnívoros } \\
\hline Cmon & 18,85 & & & \\
\hline Alac & 3,75 & & & \\
\hline Ilab & 2,71 & 2,71 & $\begin{array}{l}\text { ARBo (negativo); boca subterminal, dentição pouco } \\
\text { desenvolvida (Ilab + Aaff) ou ausente (Sins) }\end{array}$ & Nectobentônicos insetívoros \\
\hline Aaff & 13,54 & 18,85 & $\begin{array}{l}\text { Ilab + Aaff: ARO, CRPe, ArRNC, ArRNPet } \\
\text { (positivos); ICC, ARC, ICPe (negativos) }\end{array}$ & Nectobentônicos detritívoros \\
\hline Sins & 5,31 & \multirow{3}{*}{27,08} & Sins: corpo fusiforme & \\
\hline Mint & 3,54 & & \multirow{2}{*}{$\begin{array}{l}\text { ICC, ARC, ICPe; CRC, ARO, CRPe, ARBo (nega- } \\
\text { tivos); boca terminal, dentes multicuspidados }\end{array}$} & \multirow[t]{2}{*}{ Nectônicos onívoros } \\
\hline Aalt & 23,54 & & & \\
\hline Snas & 4,69 & 4,69 & $\begin{array}{l}\text { CRC; ARO, ICC, ARC, ARBo (negativos); corpo } \\
\text { fusiforme, boca subterminal, dentes multicuspi- } \\
\text { dados }\end{array}$ & Nectônicos herbívoros \\
\hline
\end{tabular}


squamosissimus e C. monoculus apresentam, além disso, nadadeiras peitorais de grande área, bem como pedúnculos caudais relativamente longos. Tanto a primeira (Agostinho \& Júlio-Jr. 1999, Bennemann et al. 2000, Agostinho et al. 2003) quanto a segunda (Agostinho \& Júlio-Jr. 1999, Agostinho et al. 2003) têm como comportamento de captura a perseguição ativa da presa à meia água ou rastreando rente ao fundo. Assim, pedúnculos caudais longos auxiliariam em um nado eficiente e constante. Nadadeiras peitorais contribuiriam para o posicionamento do peixe antes do ataque à presa. Os olhos tendendo à posição dorsal na cabeça estariam relacionados à uma tendência de ambas as espécies ocuparem estratos médios e inferiores da coluna d'água, ao passo que A. lacustris tende a ocupar estratos mais superficiais (Almeida et al. 1997, Bennemann et al. 2000) e não teria a mesma eficiência natatória que as duas outras devido ao pedúnculo mais curto, de acordo com as atribuições ecomorfológicas.

Entre as espécies bentófagas incluem uma insetívora e duas detritívoras, somando juntos cerca de $22 \%$ dos indivíduos coletados. Entre as espécies típicas de fundo não houve um padrão uniforme quanto à forma do corpo, havendo maior semelhança quanto à orientação, tamanho e dentição da boca. Iheringichthys labrosus e A. affinis apresentaram como atributos em comum pedúnculo longo e nadadeiras peitorais e caudais de grande área, atributos apresentados na literatura como freqüientemente relacionados a espécies bentônicas (Gatz 1979b, Watson \& Balon 1984, Matthews 1998). Se por um lado estes atributos estão relacionados a peixes com boa capacidade natatória, por outro, se relacionam a peixes de hábitos bentônicos, sendo comumente observados em espécies que vivem sobre o substrato e/ou em ambientes de corredeiras, estando de acordo com os dados biológicos da dieta das duas espécies (Bennemann et al. 2000, Shibatta et al. 2002, Fugi et al. 2001). Steindachnerina insculpta apresentou posição intermediária na ACP entre espécies de corpos robustos e espécies de corpos lateralmente comprimidos e altos. As três espécies apresentaram em comum boca subterminal, indicando que se alimentam próximo ou sobre o substrato. A dentição é pouco desenvolvida, sendo composta por placas dentígeras em I. labrosus, dentes incisivos somente na porção superior da boca em A. affinis ou estando completamente ausente em $S$. insculpta. A dentição pouco desenvolvida ou ausente parece ser relacionada à uma dieta micrófaga, caso de A. affinis e S. insculpta (Agostinho \& Júlio-Jr. 1999, Fugi et al. 2001), detritívoros, ou baseada em organismos de pouca mobilidade, caso de I. labrosus, que se alimentou principalmente de larvas de Chironomidade e, em menor proporção, detrito, ingerido em conjunto com o sedimento. Entretanto, como há espécies de peixes (p. ex., alguns Cyprinidae) que não possuem dentes na cavidade bucal, mas que são predadores piscívoros (Sibbing et al. 1994, Lowe-McConnell 1999), não podemos dizer que a microfagia esteja obrigatoriamente relacionada à dentição pouco desenvolvida.

O grupo formado por $M$. intermedia e A. altiparanae foi classificado como onívoro, representando $27 \%$ do total de indivíduos coletados. Apresentaram como atributos em comum corpo lateralmente comprimido e relativamente alto, com bocas terminais e dentes multicuspidados, indicando preferência por ambientes de águas calmas, como nas margens, e capacidade de realizar deslocamentos verticais. Moenkhausia intermedia, entretanto, tende a freqüentar regiões pelágicas em cardumes, como observado no rio Tibagi (Bennemann et al. 2000, Bennemann \& Shibatta 2002) onde se alimentam de zooplâncton. Apesar de ambas serem consideradas espécies onívoras segundo dados da literatura, sua morfologia reflete mais a afinidade taxonômica que o grupo trófico a que pertencem. Segundo Horn (1998), espécies de peixes onívoras possuem grande variação morfológica, o que provavelmente se relaciona à falta de especializações que caracterizam membros deste grupo trófico.

A única espécie herbívora foi $S$. nasutus, representando $4,7 \%$ dos indivíduos coletados. Na ACP, apresentou atributos relacionados, segundo a ecomorfologia, a peixes de águas rápidas, bons nadadores e que se alimentam de itens de pequeno porte (Agostinho \& Júlio-Jr. 1999), migradora (Shibatta et al. 2002), confirmando dados conhecidos para a espécie. A boca, pequena e subterminal, indica que a espécie é micrófaga, podendo capturar itens à meia-água ou ao fundo, alimentando-se de plantas. A dentição constituída por dentes multicuspidados relaciona-se ao corte de folhas e é, segundo Winemiller (1992), associada tanto à herbivoria quanto à onivoria. A forma do corpo é mais relacionada ao grupo taxonômico a que pertence que propriamente ao grupo trófico, pois espécies de outras famílias que incluem grandes proporções de matéria vegetal em sua dieta (Colosoma sp. e Brycon sp.), apresentam forma geral do corpo diferentes.

Araújo-Lima et al. (1995), em uma revisão sobre padrões tróficos de assembléias de peixes de riachos, rios e reservatórios brasileiros, afirmam que espécies piscívoras, detritívoras e onívoras costumam estar entre as dominantes em reservatórios de usinas hidroelétricas, um padrão confirmado no presente estudo. Em termos de dominância destacaram-se os piscívoros $(29,9 \%)$, onívoros $(27,1 \%)$ e detritívoros (18,8\%). Cichla monoculus e P. squamosissimus, reunidas na guilda trófica dos nectônicos carnívoros, apresentaram-se bastante semelhantes nas análises ecomorfológicas e tróficas, podendo-se dizer que ambas estão utilizando o habitat de maneira semelhante. Entretanto, conforme Bennemann et al. (2000) e Smith et al. (2003) observaram, respectivamente, no baixo Tibagi e em reservatórios no rio Tietê, $P$. squamosissimus e $C$. monoculus estão temporalmente segregados no uso de recursos tróficos, com $P$. squamosissimus forrageando preferencialmente durante o crepúsculo e à noite e $C$. monoculus preferindo o período diurno. Assim, apesar de ambas forragearem na zona litoral e utilizarem essencialmente os mesmos tipos de hábitats (pelágico e marginal), o fazem de modo alternado. Ademais, apesar de observações recentes quanto à diminuição do número de exemplares de A. altiparanae no trecho estudado, desconhece-se até o momento a abundância das populações de organismos comumente predados por ambas as espécies no reservatório, impossibilitando, pois, afirmar a existência ou não de competição. Seriam necessários estudos mais aprofundados sobre a dinâmica trófica e populacional do reservatório, permitindo a detecção de eventuais alterações ao longo do tempo. Até o presente é possível afirmar que a competição por alimentos, caso exista, é indireta e minimizada pelo menos pela segregação temporal. Por fim, de acordo com o observado neste estudo, a ecomorfologia revelou-se uma metodologia adequada na descrição de aspectos ecológicos das espécies, refletindo, preferências alimentares e de uso do habitat.

\section{Agradecimentos}

Agradecemos Lilian Casatti e Reinaldo José de Castro pelas valiosas sugestões e correções da Dissertação de Mestrado que originou este manuscrito; Oscar A. Shibatta, pela identificação das espécies e curadoria dos exemplares utilizados; Mário Luis Orsi, do Museu de Zoologia da Universidade Estadual de Londrina (MZUEL), responsável pelas coletas dos peixes; a Edson Santana e Aparecido de Souza, técnicos do MZUEL, que efetuaram as coletas; ao IBAMA pela permissão de coleta ( $n^{\circ}$ 083/2003); e ao Convênio FAUEL/UEL/ DUKE ENERGY, pelo financiamento do projeto que proporcionou o estudo; a CAPES pela Bolsa concedida.

\section{Referências bibliográficas}

ADDINSOFT. 2004. XLSTAT- Pro 7.5 - Statistical Software for MS Excel.

AGOSTINHO, A.A. \& JÚLIO-JR., H.F. 1999. Peixes da bacia do alto rio Paraná. In Estudos ecológicos de comunidades de peixes tropicais (R.H. Lowe-McConnell, ed.). Edusp, São Paulo, p.374-400.

AGOSTINHO, A.A., MIRANDA, L. E., BINI, L.M., GOMES, L.C., THOMAZ, S.M. \& SUZUKI, H.I. 1999. Patterns of colonization in neotropical reservoirs, and prognoses on aging. In Theoretical reservoir ecol- 
ogy and its applications (J. G. Tundisi \& M. Straskraba, eds.). International Institute of Ecology/Backhuys Publishers, São Carlos, p.227-265.

AGOSTINHO, A.A., GOMES, L.C. \& JÚLIO-JR., H.F. 2003. Relações entre macrófitas aquáticas e fauna de peixes. In Ecologia e manejo de macrófitas aquáticas (S.M. Thomaz \& L.M. Bini, eds.). Eduem, Maringá, p.261-276.

ALMEIDA, V.L.L., HAHN, N.S. \& VAZZOLER, A.E.A.M. 1997. Feeding patterns in five predatory fishes of the high Paraná river floodplain (PR, Brazil). Ecol. Fresh. Fish 6:123-133.

ARAÚJO-LIMA, C.A.R.M., AGOSTINHO, A.A. \& FABRÉ, N.N. 1995. Trophic aspects of fish communities in brazilian rivers and reservoirs. In Limnology in Brazil (J.G. Tundisi, C.E.M. Bicudo, T. Matsumura-Tundisi, eds.). ABC/SBL, Rio de Janeiro, p.105-136.

BALON, E.K., CRAWFORD, S.S. \& LELEK, A. 1986. Fish communities of the upper Danube river (Germany, Austria) prior to the new Rhein-MainDonau connection. Env. Biol. Fish. 15(4):243-271.

BARRELLA, W., BEAUMORD, A.C. \& PETRERE-JR, M. 1994. Comparison between the fish communities of Manso river (MT) and Jacaré Pepira river (SP), Brazil. Acta Biol.Venez. 15(2):1-15.

BEAUMORD, A.C. \& PETRERE-JR., M. 1994. Fish communities of Manso river, Chapada dos Guimarães, MT, Brasil. Acta Biol. Venez. 15(2):21-35.

BENNEMANN, S.T. \& SHIBATTA, O.A. 2002. Dinâmica de uma assembléia de peixes do rio Tibagi. In A bacia do rio Tibagi (M.E. Medri, E. Bianchini, Shibatta O.A. \& J.A. Pimenta, eds.). UEL, Londrina, p.433-442.

BENNEMANN, S.T., SHIBATTA, O.A. \& GARAVELLO, J.C. 2000. Peixes do rio Tibagi: uma abordagem ecológica. EDUEL, Londrina.

CLIFTON, K.B \& MOTTA, P. J. 1998. Feeding morphology, diet, and ecomorphological relationships among five Caribbean Labrids (Teleostei, Labridae). Copeia 4:953-966.

DOUGLAS, M.E. \& MATTHEWS, W.J. 1992. Does morphology predicts ecology? Hypothesis testing within a fish assemblage. Oikos 65:213-224.

DUKE ENERGY. 2003. Peixes do rio Paranapanema. Editora Horizonte Geográfico, São Paulo.

FELLEY, J.D. 1984. Multivariate identification of morphological - environmental relationships within the Cyprinidae (Pisces). Copeia (2):442-455.

FREIRE, A.G. \& AGOSTINHO, A.A. 2001. Ecomorfologia de oito espécies dominantes da ictiofauna do reservatório de Itaipu (Paraná/Brasil). Acta Limnol. Bras. 13(1):1-9.

FUGI, R., AGOSTINHO, A.A. \& HAHN, N.S. 2001. Trophic morphology of five benthic-feeding fish species of a tropical floodplain. Rev. Bras. Biol. 61(1):27-33.

GATZ JR., A.J. 1979a. Community organization in fishes as indicated by morphological features. Ecology 60(4):711-718.

GATZ JR., A.J. 1979b. Ecological morphology of freshwater stream fishes. Tulane Stud. Zool. Bot. 21(2):91-124.

HAMMER, Ø., HARPER, D.A.T. \& RYAN, P.D. 2004. PaST-Palaeontological Statistics, version 1.32. Disponível em http://folk.uio.no/ohammer/past/ (consulta em 10/09/2005).

HOFFMANN, A.C., ORSI, M.L. \& SHIBATTA, O.A. 2005. Diversidade de peixes do reservatório da UHE Escola Engenharia Mackenzie (Capivara), rio Paranapanema, bacia do alto rio Paraná, Brasil, e a importância dos grandes tributários na sua manutenção. Iheringia Sér. Zool. 95(3): 319-325.

HORN, M.H. 1998. Feeding and digestion. In The physiology of fishes (D. H Evans ed.). $2^{\text {nd }}$ ed. CRC Press, Boca Raton, p.43-64.

HUGUENY, B. \& POUILLY, M. 1999. Morphological correlates of diet in an assemblage of West African freshwater fishes. J. Fish Biol. 54:1310-1325.

HYSLOP, J. 1980. Stomach contents analysis - a review of methods and their application. J. Fish Biol. 17:411-429.

KAWAKAMI, E. \& VAZZOLER, G. 1980. Método gráfico e estimativa de índice alimentar aplicado no estudo de alimentação de peixes. Bol. Inst. Oceanogr. 29(2)205-207.

LOWE-McCONNELL, R.H. 1999. Estudos ecológicos de comunidades de peixes tropicais. Edusp, São Paulo.

MAACK, R. 1981. Geografia física do estado do Paraná. Rio de Janeiro/Curitiba, José Olympio/Secretaria da Cultura e do Esporte do Governo do Estado do Paraná.

MAHON, R. 1984. Divergent structure in fish taxocenes of north temperate streams. Can. J. Fish Aquat. Sci. 41:330-350.

MATTHEWS, W.J. 1998. Patterns in freshwater fish ecology. Kluwer Academic Publishers, Dordrecht.

MÉRONA, B., Santos, G.M. \& Almeida, R.G. 2001. Short term effects of Tucuruí dam (Amazonia, Brazil) on the trophic organization of fish communities. Env. Biol. Fish. 60:375-392.

MINISTÉRIO DE MINAS E ENERGIA/COMITÊ COORDENADOR DO PLANEJAMENTO DE EXPANSÃO DOS SISTEMAS ELÉTRICOS (CCPE). 2002. Plano decenal de expansão 2003-2012. Disponível em http://www.ccpe.gov.br (consulta em 10/09/2005).

NORTON, S.F., LUCZKOVICH, J.J. \& MOTTA, P.J. 1995. The role of ecomorphological studies in the comparative biology of fishes. Env. Biol. Fish. 44(1-2):287-304.

PIANKA, E.R. 2000. Evolutionary ecology. $6^{\text {th }}$ ed. Addison Wesley Longman, San Francisco.

POUILLY, M, LINO, F., BRETENOUX, J.-G. \& ROSALES, C. 2003. Dietary-morphological relationships in a fish assemblage of the bolivian amazonian floodplain. J. Fish Biol. 62:1137-1158.

SIBBING, F.A., NAGELKERKE, L.A.J. \& OSSE, J.W.N. 1994. Ecomorphology as a tool in fisheries: identification and ecotyping of lake Tana barbs (Barbus intermedius complex), Ethiopia. Neth. J. Agric. Sci. 42(1):77-85.

SHIBATTA, O.A., ORSI, M.L., BENNEMANN. S.T. \& SILVA-SOUZA, A.T. 2002. Diversidade e distribuição de peixes na bacia do rio Tibagi. In A bacia do rio Tibagi (M.E. Medri, E. Bianchini, O.A. Shibatta \& J.A. Pimenta, eds.). UEL, Londrina, p.403-423.

SMITH, W.S., PEREIRA, C.C.G.F., ESPÍNDOLA, E.L.G. \& ROCHA, O. 2003. A importância da zona litoral para a disponibilidade de recursos alimentares à comunidade de peixes em reservatórios. In Ecótonos nas interfaces dos ecossistemas aquáticos (R. Henry, org.). RiMa, São Carlos, p.233-248.

VALENTIN, J.L. 2000. Ecologia numérica: uma introdução à análise multivariada de dados ecológicos. Editora Interciência, Rio de Janeiro.

VAZZOLER, A.E.A.M. 1996. Biologia da reprodução de peixes teleósteos: teoria e prática. Eduem/SBI/CNPq/Nupélia, Maringá.

WATSON, D.J. \& BALON, E.K. 1984. Ecomorphological analysis of fish taxocenes in rainforest streams of northern Borneo. J. Fish Biol. 25:371-384.

WIKRAMANAYAKE, E.D. 1990. Ecomorphology and biogeography of a tropical stream fish assemblage: evolution of assemblage structure. Ecology 71(5):1756-1764.

WINEMILLER, K.O. 1992. Ecomorphology of freshwater fishes. Res. Explor. $8(3): 308-327$.

WINEMILLER, K.O., KELSO-WINEMILLER, L.C. \& BRENKERT, A.L. 1995. Ecomorphological diversification and convergence in fluvial cichlid fishes. Env. Biol. Fish. 44(1-2):235-261. 